\title{
Quel avenir pour l'élevage dans le bassin cotonnier de l'Ouest du Burkina Faso ? Dynamiques agro-pastorales et recompositions territoriales
}

\author{
Alexis Gonin, Bernard Tallet
}

L'Ouest du Burkina Faso connait depuis un demi-siècle des mutations spatiales majeures, qui ont des répercussions importantes sur les deux principales activités rurales régionales, l'agriculture et l'élevage. Cette région appartient à la zone écologique des savanes, à l'articulation entre des milieux sahéliens au nord, où la pluviométrie n'excède pas $700 \mathrm{~mm}$ par an, et des milieux soudaniens au sud, où la pluviométrie atteint en moyenne $1000 \mathrm{~mm}$ par an. Avant les années 1960, les densités dans la région excédaient rarement 10 habitants $/ \mathrm{km}^{2}$, tandis que de vastes zones étaient quasiment vides, les densités n'atteignant pas 1 habitant $/ \mathrm{km}^{2}$ [Savonnet, 1968]. L’agro-élevage villageois était très peu développé ; les troupeaux peuls transhumaient essentiellement dans la zone nord (plaine du Gondo-Sourou). À partir du début des années 1970, un front pionnier, alimenté par des migrations d'agriculteurs mossis venus du plateau central du Burkina Faso, transforme radicalement le visage de la région. La forêt, jusqu'alors dominante dans le paysage, est défrichée, laissant place à un espace quasi exclusivement dominé par les champs. L'Ouest devient le principal bassin cotonnier du pays, et par conséquent une région stratégique sur le plan économique, le coton étant le premier produit d'exportation du Burkina. Les densités, sauf dans le sud de la région, atteignent couramment 60 habitants $/ \mathrm{km}^{2}{ }^{1}$. Les Mossi sont désormais majoritaires dans tous les villages, loin devant les ethnies autochtones (Bobo, Bwa, Samo, Sénoufo) et les éleveurs peuls. La dynamique pionnière s'essouffle dès les années 1990, tout l'espace disponible ayant été mis en valeur (sauf dans le sud). Mais les mutations qu'elle a provoquées dans les paysages, le peuplement, la mise en valeur de la région sont spectaculaires. L'organisation spatiale de l'élevage en est notamment complètement bouleversée. Ces évolutions ultérieures au front pionnier, réclament de prendre un peu plus de recul temporel : il s'agit de resituer la vingtaine d'années du front pionnier dans un temps plus long [Tallet, 2007].

1. Source : Institut National de la Statistique et de la Démographie (INSD), Burkina Faso. 
Pour cela, des enquêtes de terrain ${ }^{2}$ ont été couplées à des recherches dans les archives. Le propos de cet article est d'en interpréter les résultats dans le but de comprendre les transformations du rapport des éleveurs de l'Ouest à leur espace sur un demi-siècle et d'éclairer le sens possible des évolutions pastorales à venir. Avec le passage «du vide au plein » [Paré, Tallet, 1999], l'espace est devenu une ressource rare pour les agriculteurs et les éleveurs. Il fait l'objet de tentatives de délimitation et d'appropriation qui sont sources de tensions sociales, parfois de conflits. À cet égard, l'Ouest du Burkina ne diffère pas d'autres régions soudanosahéliennes où les densités de population sont devenues très fortes, provoquant un accroissement de la concurrence spatiale entre agricultures et élevages (pays Sereer au Sénégal [Lericollais, 1999], sud du Tchad [Magrin, 2001], nord du Cameroun [Seignobos, Thys, 1998]). Il s'agit de montrer que la compétition spatiale est le reflet d'une compétition socio-économique. Dans les villages de l'Ouest burkinabé, une nouvelle hiérarchie socio-économique émerge, qui transcende les catégories ethniques habituelles. Des rapports de force nouveaux se dessinent : en fonction de leur résolution, différents scénarios peuvent être établis pour l'avenir de l'élevage dans la région.

\section{Dynamique pionnière et mutations des espaces pastoraux}

À l'échelle régionale et dans une perspective temporelle longue, la zone cotonnière apparait comme un lieu de passage, de transit au sein de territoires pastoraux s'étendant du nord au sud sur quelques centaines de kilomètres, utilisant au mieux la complémentarité climatique de la zone soudano-sahélienne. La mobilité est le ressort principal de la dynamique pastorale. Ce schéma d'utilisation de l'espace régional semble remis en cause par les transformations des systèmes d'activités au sein du bassin cotonnier qui ont bouleversé les modes de gestion de l'espace.

\section{L'espace régional du pastoralisme peul transhumant}

Avec l'interdiction de la traite esclavagiste par l'administration coloniale française au début du Xx' siècle, les Peuls installés dans les chefferies de Barani et Dokuy, au nord-ouest du Burkina, ont dû abandonner leur système économique fondé sur l'exploitation d'une main-d'œuvre asservie (les rimaïbé) et sur la razzia des villages bobos et bwas voisins [Diallo, 1997]. Retrouvant leurs pratiques du XIX siècle, ils se sont alors de nouveau tournés vers un élevage transhumant. Les troupeaux ont rapidement augmenté, notamment grâce aux progrès vétérinaires introduits par les politiques coloniales. Dans les années 1940, il a été nécessaire de faire transhumer les zébus en saison sèche au-delà des pâturages de Barani et

2. Les enquêtes de terrain ont été menées dans deux départements : Padéma, à quatre-vingts kilomètres au nord de Bobo-Dioulasso, la principale ville de l'Ouest, et Samorogouan, à soixante kilomètres à l'ouest, en 2008 et en 2009 dans le cadre d'une mission de recherche pilotée par l'UMR Prodig (suivi du projet pilote de sécurisation foncière en zone cotonnière) et d'un mémoire de master 2 de l'université Paris 1 . Près d'une centaine d'entretiens ont été menés auprès d'agriculteurs, d'éleveurs, de responsables administratifs, politiques, syndicaux. Ces enquêtes sont prolongées par un travail de thèse. 
Carte - Les mouvements de transhumance dans l'Ouest du Burkina Faso

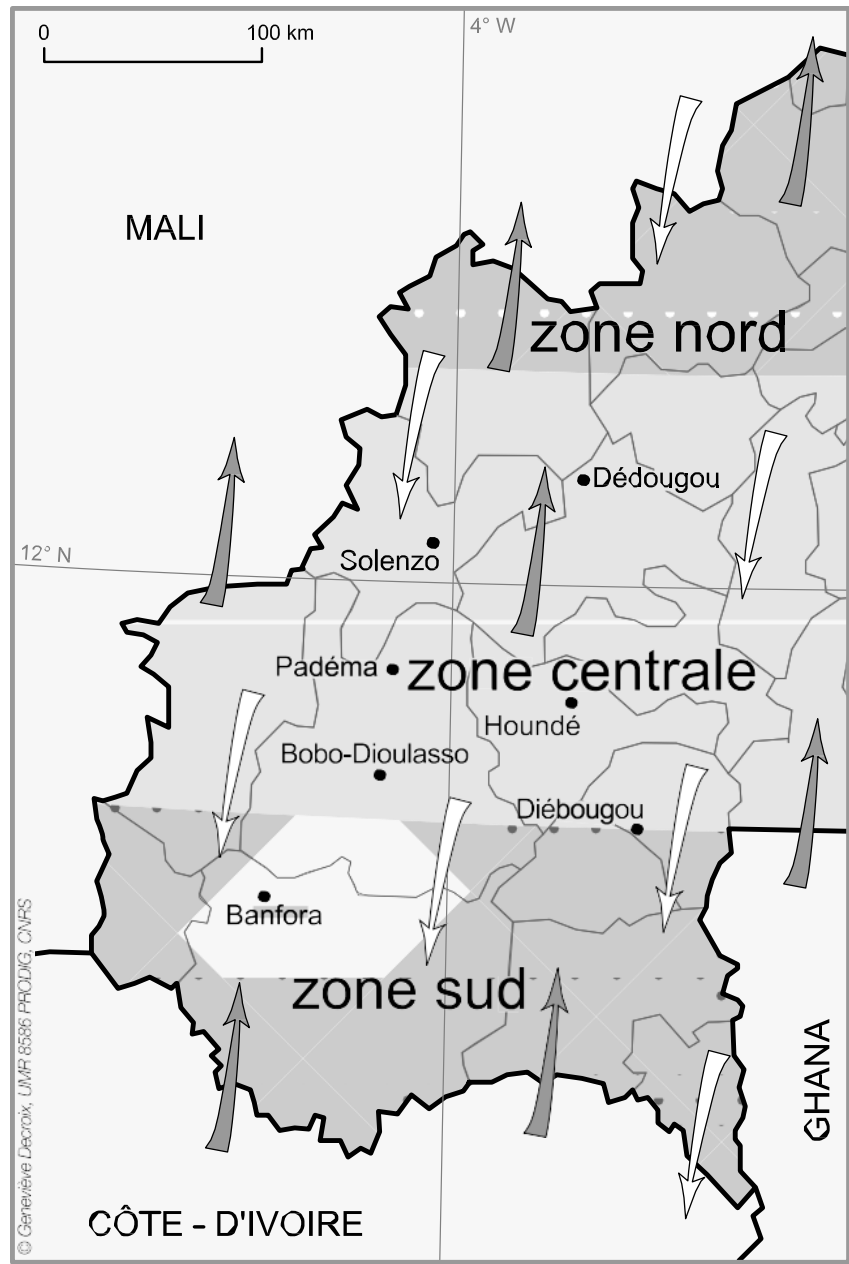

présence des troupeaux durant la saison des pluies

passage des troupeaux lors des transhumances

accueil des troupeaux durant la saison sèche

transhumance (novembre-décembre)

transhumance (mai-juin) 
Dokuy. Les ressources fourragères de la vallée du Mouhoun et de la plaine du Sourou ont été utilisées par les Peuls. À partir des années 1960, les parcours de transhumance s'allongent vers le sud en direction de Bobo-Dioulasso en suivant la vallée du Mouhoun [Benoit, 1979]. Se met alors en place le système transhumant peul qui perdure tant bien que mal aujourd'hui, fondé sur la mise en complémentarité par la mobilité des troupeaux de trois grandes zones bioclimatiques (carte 1, p. 97) :

- Une zone nord sahélienne : l'activité pastorale y est dominante depuis au moins le XIX siècle, même si elle ne permet plus le maintien de l'ensemble des troupeaux tout au long de l'année ; espace de concentration des troupeaux pendant la saison des pluies, elle ne conserve qu'un noyau d'élevage en saison sèche. Mais l'élevage y apparait de plus en plus concurrencé par la progression des défrichements agricoles.

- Une zone centrale, marquée par l'extension des espaces agricoles en liaison avec la dynamique cotonnière, voit sa fonction de zone de transit menacée. S'il existe encore des espaces proprement pastoraux, ceux-ci perdent leur accessibilité et sont l'objet d'une pression foncière croissante.

- La zone sud, soudanienne, devient la seule zone d'accueil possible des troupeaux en saison sèche. À partir des années 1970, les défrichements agricoles, en réduisant les surfaces boisées et en étendant les clairières cultivées, entrainent la disparition progressive de la trypanosomiase, maladie mortelle pour les zébus. Les troupeaux peuvent désormais séjourner plus longtemps dans cette zone ; en même temps ils y subissent de plus en plus fortement la concurrence de l'agriculture.

Cette utilisation des complémentarités régionales est une pratique courante, observée ailleurs en Afrique de l'Ouest [Boutrais, 1988] et dont l'efficience économique et écologique a été plusieurs fois démontrée [Homewood, 2008 ; Jullien, 2006 ; Marty, Bonnet, Guibert, 2006]. Mais si la dimension régionale de l'élevage est évidente, elle se trouve confrontée dans l'Ouest burkinabé à des dynamiques internes à chaque zone qui, conjuguées, aboutissent à la difficulté voire l'impossibilité de maintenir les pratiques pastorales mises en place dans les années 1960. En effet, à grande échelle géographique, la logique de la transhumance peule implique que le déplacement quotidien des troupeaux soit l'occasion de pâturer. Avant la mise en place du front pionnier, les espaces de confins, à la périphérie des terroirs villageois, offraient de grands pâturages. Pour y guider les troupeaux, les bergers ont instauré des relations avec des Peuls installés en campement à proximité des villages ou avec des agriculteurs autochtones ${ }^{3}$. Les « logeurs » (djatigui en dioula) informent toujours les bergers peuls au cours de leur déplacement de la disponibilité et de la qualité des pâturages locaux. Ces pâturages ont été progressivement mis en culture, notamment dans la zone centrale (bassin cotonnier) : les troupeaux transhumants peinent à présent à trouver des ressources

3. En échange de leurs services, les agriculteurs villageois recevaient du lait lors du passage des Peuls, les troupeaux fumaient leurs champs. 
fourragères lors de leurs déplacements. Les effets à long terme de la dynamique pionnière exercent une menace croissante sur le système transhumant mis en place il y a cinquante ans.

\section{Le développement del'agro-élevage et la réduction des mobilités pastorales}

Avant le début des années 1970, marquées dans l'Ouest burkinabé par l'arrivée massive de migrants et l'ouverture du front pionnier, l'élevage n'était présent que sporadiquement dans les savanes sahélo-soudaniennes ${ }^{4}$. Le beurre de karité, obtenu grâce à l'arbre du même nom, permettait de pallier l'insuffisance de matières grasses d'origine animale dans le régime alimentaire des agriculteurs. Les très importants vestiges des parcs à karité en pays bobo, à proximité des villages, sont ainsi un indice de l'absence d'élevage dans la région jusqu'à une date récente [Pélissier, 1978]. L'élevage sédentaire présente un premier développement dans les années 1970, avant de connaitre un essor accéléré dans la décennie suivante. Ce développement provient pour une part de l'installation des migrants peuls venus du nord sahélien dans l'Ouest du Burkina. Cette descente vers le sud, initiée dès les années 1960, s'inscrit dans un mouvement général à toute l'Afrique de l'Ouest de migration des Peuls vers les régions soudaniennes [Boutrais, 1994]. Elle est alors encouragée par la recherche de nouveaux pâturages pour des troupeaux de plus en plus importants, la disparition progressive de la trypanosomiase sous l'effet des défrichements et l'absence de taxe sur l'élevage dans des pays comme la Côte d'Ivoire [Bassett, 1986 ; Bernardet, 1999]. Cette translation vers le sud a été accélérée par les sécheresses sahéliennes des années 1970 et 1980. Une fois installés plus au sud, les Peuls ont tendance à réduire les déplacements de leurs troupeaux, qui peuvent trouver des pâturages de bonne qualité à proximité du campement une grande partie de l'année. Ces éleveurs peuvent alors cultiver quelques hectares de maïs ou de sorgho : ils deviennent des agro-pasteurs.

La deuxième origine de l'élevage sédentaire dans l'Ouest est la diffusion de la culture attelée auprès des agriculteurs autochtones et des migrants mossis. Le premier contact des agriculteurs avec les bovins provient de l'acquisition d'une paire de bœufs de trait. Progressivement, une large partie des exploitations se dotent d'un attelage. La charrue tirée par des bœufs permet d'emblaver de plus grandes surfaces : c'est bien la diffusion de la culture attelée qui provoque une «course à la terre », le défrichement de la brousse et la rapide saturation de tout l'espace disponible par les champs dans le bassin cotonnier [Tersiguel, 1995]. Les agriculteurs qui réussissent, grâce à la culture attelée, à agrandir leurs surfaces plantées en coton, parviennent à dégager un surplus financier à l'issue des bonnes campagnes agricoles. Pour épargner ce surplus financier, ils l'investissent dans des bovins, et développent peu à peu des troupeaux qui peuvent

4. Seules les communautés Bwa [Savonnet, 1959] et Sénoufo [Hoffmann, 1985] entretenaient de petits troupeaux qui faisaient partie intégrante de leur système agricole. 
Carte - La mise en territoire des espaces pastoraux dans le département de Padéma

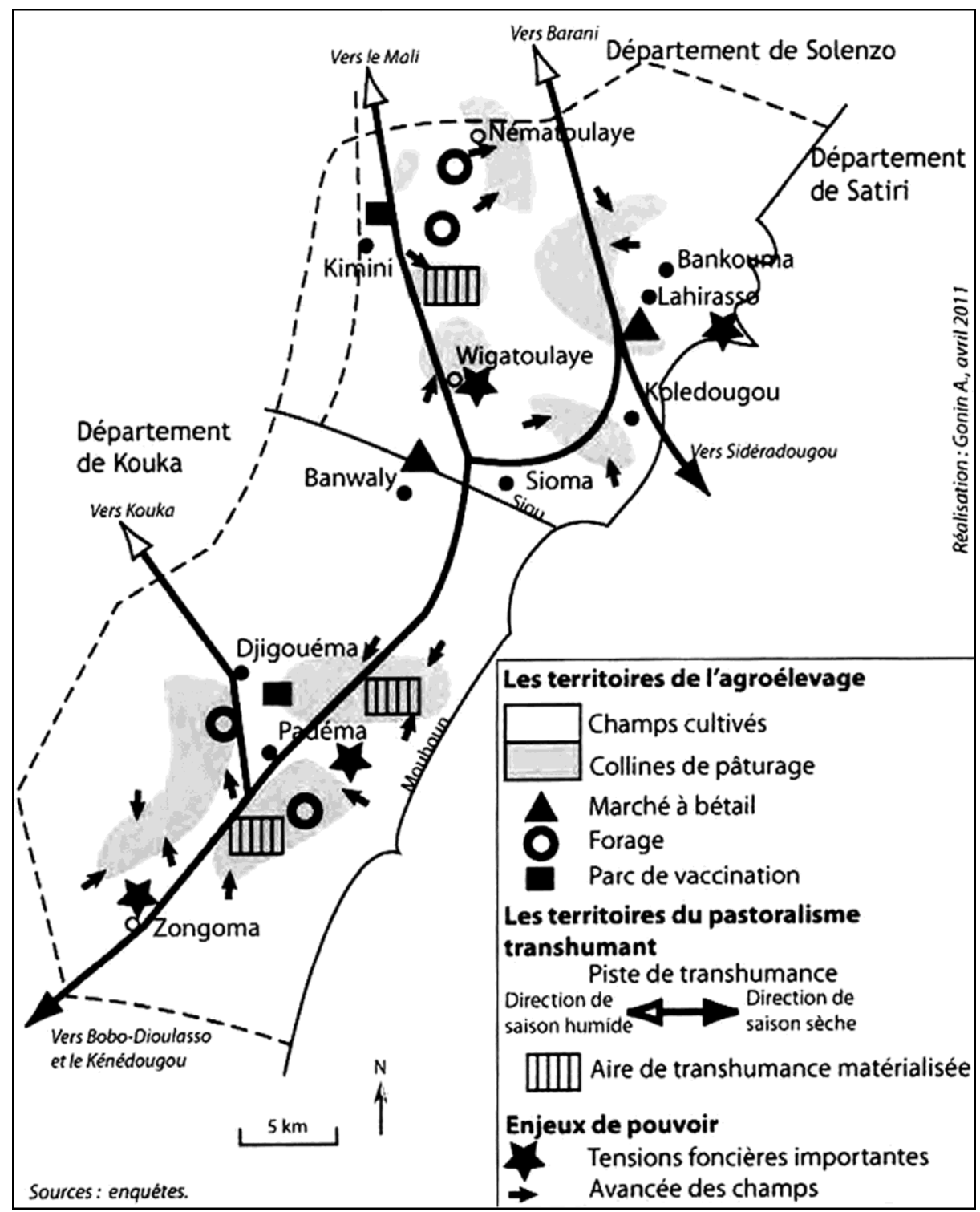


être très importants (jusqu'à trois cents têtes pour les plus grands, soit beaucoup plus que la très grande majorité des troupeaux des Peuls). Ces troupeaux sont trop importants pour trouver suffisamment de fourrage dans les pâturages locaux en saison sèche : ils descendent alors vers le nord de la Côte d'Ivoire. Ce processus qui conduit à la diversification des activités dans les exploitations agricoles (dont le chef peut être qualifié d'agro-éleveur) est bien connu et a été décrit plusieurs fois, par exemple dans le sud du Tchad [Magrin, 2001] ou en pays sereer au Sénégal [Lericollais, 1999 ; Cesaro, Magrin, Ninot, 2010]. Contrairement au pays sereer, où l'intégration de l'élevage à l'agriculture au sein des exploitations a été réalisée de longue date, les agriculteurs de l'Ouest burkinabé ne parviennent pas à réellement augmenter leurs rendements grâce à la fumure animale. Les agronomes [Dongmo et al., 2007 ; Vall, Dugué, Blanchard, 2006] ont pourtant démontré qu'il existe de nombreuses complémentarités à mettre en œuvre entre élevage et agriculture, à l'échelle de l'exploitation ou du terroir villageois (fumure animale pour améliorer la fertilité des champs, utilisation des résidus de récolte comme fourrage, traction animale). Or, rares sont les agro-éleveurs ou les agro-pasteurs ${ }^{5}$ de l'Ouest burkinabé qui mettent pleinement en valeur la complémentarité agronomique élevage/agriculture. La dynamique pionnière aurait pu être l'occasion d'un développement intégré de l'agriculture et de l'élevage. Chacune des activités aurait pu se renforcer l'une l'autre, conduisant ainsi à une intensification des productions végétales et animales. L'espace n'aurait pas été saturé. Mais la dynamique pionnière a dans les faits été l'occasion d'un développement parallèle de l'agriculture et de l'élevage, chacune des deux activités restant pratiquée, pour l'essentiel, selon un mode de production extensif très consommateur d'espace. Historiquement, la dynamique pionnière a ainsi conduit à une saturation de l'espace, dont les effets se font encore sentir aujourd'hui et restent un déterminant majeur des évolutions futures de la région.

\section{Une « mise en territoire » des espaces pastoraux}

Les différents acteurs de l'élevage ont aujourd'hui, et dans les années à venir, à gérer un héritage essentiel de l'époque du front pionnier : la saturation de l'espace.

\section{Des espaces ouverts aux territoires finis}

Avant la fin du front pionnier, le pastoralisme se pratiquait sur des espaces ouverts. Les villages étaient séparés par de véritables no man's land; la brousse paraissait infinie. Elle n'était exploitée que par des chasseurs occasionnels et restait largement inexplorée une grande partie de l'année. Ces périphéries faiblement appropriées (aux sens juridique, politique, social et symbolique) par les

5. Les agro-éleveurs sont à l'origine des agriculteurs qui ont diversifié leur exploitation pour pratiquer également l'élevage, les agro-pasteurs sont à l'origine des pasteurs qui ont diversifié leur exploitation pour pratiquer également de l'agriculture [Augusseau, 2008]. 
autochtones étaient les terrains de parcours privilégiés des rares pasteurs transhumants. Plus tard, lors de l'installation des pasteurs peuls et de l'acquisition de troupeaux par des agro-éleveurs, les larges parties de la brousse qui n'étaient pas encore défrichées servaient de pâturages permanents. Au fur et à mesure que le front pionnier progressait localement, la brousse périphérique a été défrichée et mise en culture. Les pâturages se sont progressivement réduits comme peau de chagrin, à l'exemple de ce que l'on peut observer dans un département comme celui de Padéma, au cœur de la zone cotonnière (carte p. 100). L'augmentation des cheptels, à la fois chez les agro-éleveurs et chez les agro-pasteurs, et l'extension des surfaces cultivées grâce à la culture attelée, renforcent la concurrence : chaque activité nécessite de plus en plus d'espace.

Pour tenter d'encadrer les pratiques pastorales et agricoles dans un contexte de concurrence, des efforts de délimitation des espaces ont été menés par les villageois. Jadis, les déplacements des troupeaux dans les brousses périphériques encore abondantes n'avaient pas besoin d'être réglementés. Dans un contexte de saturation spatiale, des pistes pour les déplacements des troupeaux ont été délimitées à la peinture ou balisées dans plusieurs communes de l'Ouest. De même, la limite entre pâturages et champs a été en plusieurs lieux matérialisée ${ }^{6}$.

La concurrence entre usagers débouche souvent sur des conflits. La plupart des conflits proviennent des dégâts causés par les animaux dans les champs qui ne sont pas encore récoltés. Le mécanisme de ces conflits a été bien étudié dans le nord de la Côte d'Ivoire [Bassett, 1988]. Un arrangement financier à l'amiable entre la victime et le propriétaire des bêtes incriminées est le plus souvent trouvé. Mais, en l'absence d'intégration territoriale réelle entre éleveurs et agriculteurs, les conflits dégénèrent souvent [Gautier et al., 2005], sans aller toutefois jusqu'aux dérapages meurtriers que peuvent connaître d'autres régions [Magrin, 2001]. Un autre cas de figure observé sur le terrain est la mise en culture des pistes tracées pour le passage du bétail. Ces surfaces étroites mais très fertiles (car amendées par le passage fréquent des bovins) sont très tentantes pour les cultivateurs riverains qui ont besoin d'augmenter leur production et donc leur superficie cultivée. Un cultivateur sème sur une piste à bétail pour se l'approprier et détourner cet espace de sa fonction pastorale première. Un éleveur peut relâcher son attention sur ses bêtes pour qu'elles occasionnent des dégâts dans un champ ; la multiplication de ces dégâts au même endroit suscite peut-être l'espoir chez l'éleveur que le cultivateur abandonne son champ et que celui-ci redevienne un pâturage... Ainsi, agriculture et élevage se disputent le même espace. Cette lutte ne recoupe pas de façon simple l'opposition entre groupes, car les agro-éleveurs et les agro-pasteurs ont des intérêts partagés entre les deux activités. Ils doivent faire des arbitrages entre les pâturages pour leurs troupeaux et leurs champs, choisissant selon les cas l'un ou l'autre. Les dégâts aux cultures montrent les tentatives d'appropriation

6. Pour effectuer cette matérialisation, les villageois ont reçu l'appui de projet de développement rural, notamment le Projet de développement de l'Ouest (PDLO) de 2004 à 2009, financé en grande partie par l'Agence française de développement (AFD). 
durant la période où la concurrence spatiale est exacerbée, celle de la campagne agricole (de mai à décembre environ). Mais les luttes pour l'appropriation de l'espace ne prennent pas fin durant la saison sèche, quand les champs sont récoltés et donc potentiellement libres pour les animaux. À ce moment de l'année, la pratique qui prévalait avant le développement important de l'agro-élevage était la libre pâture : les animaux des Peuls transhumants ou sédentaires pouvaient aller librement dans les champs récoltés brouter les résidus (tiges de mil, de maïs...). En échange, le champ de l'agriculteur recevait de la fumure animale ${ }^{7}$. Désormais, les agro-éleveurs ramassent ou réservent les résidus de leur récolte pour leur propre troupeau. Ce n'est qu'une fois nettoyé que le champ est ouvert aux troupeaux étrangers. La privatisation des résidus de récolte correspond bien à une appropriation de l'espace du champ durant la saison sèche.

Deux principaux éléments ressortent des observations des pratiques agricoles et pastorales sur le terrain, comparées à d'autres régions d'Afrique de l'Ouest dans des contextes similaires [Gonin, 2011] :

- Avant le front pionnier, l'espace était flou, fait de noyaux de peuplement (villages et cultures auréolaires) et de confins (brousse). On a assisté à une disparition des confins (défrichement de la brousse) et à une délimitation plus claire de l'espace (délimitation des pistes à bétail, des pâturages, des champs, tentatives de clarification des limites de terroirs, des domaines lignagers, des parcelles...).

- Les espaces de confins occupés par la brousse sont maintenant l'objet de multiples tentatives d'appropriation foncière, politique (par les pouvoirs lignagers, coutumiers ou communaux) et économique (définition d'un usage pastoral ou agricole de l'espace). Les conflits entre acteurs locaux sont le signe le plus visible de cette appropriation de l'espace.

L'héritage du front pionnier se traduit donc aujourd'hui par la délimitation et l'appropriation de l'espace. Selon Pourtier [2005] la «mise en territoire » de l'espace se définit précisément par ces deux processus : « l'invention de droits sur l'espace, l'appropriation, individuelle ou collective, de parcelles de terre » et la délimitation de l'espace disponible («la tyrannie du trait» [2005]). Mais il ne faudrait pas conclure hâtivement de cette analyse que la «mise en territoire » de l'Ouest du Burkina est une transformation historique et radicale des espaces de l'élevage en territoires ${ }^{8}$ de l'élevage. D'abord, un territoire de l'élevage ne saurait être à vocation uniquement pastorale. Un champ est un territoire agricole lorsqu'il est cultivé (c'est bien un espace approprié et délimité) et un territoire pastoral lorsqu'il est ouvert aux troupeaux. Les limites du territoire ne le rendent pas imperméable aux autres activités. Ensuite, des espaces délimités et appropriés, donc des territoires, existaient avant la saturation spatiale. J. Gallais [1984] décrit

7. Au vu du faible nombre d'animaux avant la fin des années 1990, les apports de fumure restaient quoiqu'il en soit largement insuffisants pour constituer un amendement efficace pour l'ensemble des champs.

8. Le territoire étant défini de façon très synthétique dans le cadre de cet article comme un espace délimité et approprié. 
par exemple dans le delta intérieur du Niger des territorialités multiples et saisonnières où les différents usages des ressources (pêche, élevage, agriculture) se succèdent de façon ordonnée grâce à une appropriation de l'espace bien codifiée. Malgré ces nuances, le concept de «mise en territoire» de l'espace est d'une grande utilité pour comprendre le changement dans l'Ouest du Burkina. Il permet de mettre en lumière une organisation radicalement nouvelle de l'espace dans la région. Le caractère fini de l'espace, sa délimitation, son appropriation exclusive, donc l'exclusion de certains usages et de certains usagers, sont des enjeux devenus incontournables dans les années qui ont suivi la fermeture du front pionnier : la notion de territoire permet d'en rendre compte de façon plus efficace que celle d'espace. De plus, l'utilisation de territoire comme outil conceptuel permet d'analyser l'évolution des rapports de force entre acteurs. Ces rapports de force sont les causes explicatives des changements qui affectent le pastoralisme.

\section{" Gagnants » et " perdants "}

Les acteurs de l'élevage participent tous, plus ou moins directement, et par différents moyens, à la délimitation et à l'appropriation des espaces : ils construisent des territoires conformes à leurs intérêts. Pour discerner qui sont les différents acteurs de la "mise en territoire », les typologies souvent proposées sont construites sur des critères ethniques et les pratiques d'élevage. On aboutit ainsi à distinguer les pasteurs transhumants, de l'ethnie peule, et les éleveurs sédentaires mossis (migrants), bobos, bwas, samos... (autochtones). On peut ensuite affiner ces catégories en précisant les échelles de mobilité (grande ou petite transhumance, troupeau sédentaire...). Mais le critère ethnique, souvent mis en avant par les villageois eux-mêmes dans les entretiens, n'est pas un bon discriminant : il dissimule des oppositions entre des acteurs appartenant à la même ethnie ; il cache des alliances entre des individus d'ethnies différentes mais aux intérêts communs. Pour comprendre quels sont les acteurs capables d'orienter la « mise en territoire » en leur faveur, une typologie plus opératoire a été dressée à partir de critères socio-économiques ${ }^{9}$.

La catégorie des acteurs «gagnants » regroupe à la fois de riches agro-pasteurs peuls et de riches agro-éleveurs des ethnies autochtones et migrantes. Ces derniers ont dégagé des surplus financiers importants du coton qu'ils ont investis dans l'élevage. Les agro-pasteurs sont des Peuls qui ont réussi (grâce à une maind'œuvre familiale importante, à une bonne conduite des troupeaux lors des transhumances...) à constituer et à sauvegarder de grands troupeaux. Bien intégrés dans les villages de l'Ouest, ils ont pu acquérir des terres cultivables. Certains cultivent même quelques hectares de coton. Beaucoup de ces acteurs «gagnants » sont issus des lignages historiquement dominants du village. L'assise économique et le prestige social des riches agro-éleveurs et agro-pasteurs leur confèrent un grand

9. La typologie présentée ici est une première approche qui vise avant tout à argumenter le raisonnement. Elle devra être affinée par de nouvelles recherches sur le terrain. 
Tableau 1 - Typologie des acteurs pratiquant l'élevage en fonction de leur capacité à orienter la « mise en territoire »

\begin{tabular}{|c|c|c|}
\hline $\begin{array}{l}\text { Catégories } \\
\text { Critères }\end{array}$ & «Gagnants » & «Perdants » \\
\hline Ressources sociales & $\begin{array}{l}\text { Capital social important } \\
\text { (grands lignages, chefferies } \\
\text { traditionnelles, } \\
\text { responsabilités politiques } \\
\text { locales, responsabilités } \\
\text { dans des associations...) }\end{array}$ & $\begin{array}{l}\text { Faible capital social } \\
\text { (ethnies ou lignages } \\
\text { minoritaires, allochtones, } \\
\text { salariés...) }\end{array}$ \\
\hline Ressources économiques & $\begin{array}{l}\text { Capital économique } \\
\text { important (grandes } \\
\text { superficies, forte } \\
\text { production agricole, grands } \\
\text { troupeaux, activités } \\
\text { diversifiées...) }\end{array}$ & $\begin{array}{l}\text { Faible capital économique } \\
\text { (petites superficies, } \\
\text { mono-activité, pas de } \\
\text { bovins détenus en propre...) }\end{array}$ \\
\hline$=>$ Pouvoir local & $\begin{array}{l}\text { Pouvoir important } \\
\text { (définition des priorités, } \\
\text { influence dans les débats, } \\
\text { décisions sur les } \\
\text { aménagements...) }\end{array}$ & $\begin{array}{l}\text { Pouvoir faible (pas de } \\
\text { représentation dans les } \\
\text { instances de décisions, } \\
\text { soumission aux décisions) }\end{array}$ \\
\hline
\end{tabular}

pouvoir local. Dans tous les villages enquêtés, les postes du maire, des responsables syndicaux et des associations professionnelles sont occupés par ce type d'acteurs : il n'y a pas de dichotomie entre les pouvoirs « traditionnels » et modernes. Ils ont de ce fait un plus grand pouvoir de décision pour la délimitation des espaces du terroir villageois et la définition de leur vocation agricole ou pastorale. Les agro-éleveurs privilégient encore dans leur très grande majorité leur intérêt agricole, et notamment cotonnier. Pour cette raison, la « mise en territoire » est synonyme, dans la plupart des villages, de réduction au minimum des pâturages et d'optimisation de la taille des territoires agricoles. Les agro-pasteurs s'adaptent et participent à cette nouvelle donne territoriale. Comme les agro-éleveurs, ils envoient une grande partie de l'année leur troupeau devenu trop grand pour les pâturages locaux plus au sud, en zone soudanienne. Ils gardent quelques animaux sur place, notamment pour pratiquer l'embouche bovine. Ils cultivent ainsi leurs attaches et leur pouvoir local.

La catégorie des acteurs «perdants » regroupe à la fois des éleveurs, des agriculteurs, des agro-éleveurs et agro-pasteurs de différentes ethnies. Leur dénominateur commun est leurs faibles ressources économiques corrélées à leurs faibles ressources sociales. Il peut s'agir par exemple de pasteurs peuls qui ont perdu leur troupeau et qui s'engagent comme bergers salariés auprès de grands éleveurs tout 
en cultivant un ou deux hectares de terres. Il peut également s'agir d'agriculteurs qui pratiquent essentiellement la culture vivrière, et qui n'ont pas les moyens d'augmenter leur production (absence de culture attelée, insécurité foncière...). Les acteurs «perdants» subissent la «mise en territoire». Les petits éleveurs n'ont pas les moyens de réagir à la diminution de leur pâturage ni d'engager leur troupeau dans de longues transhumances vers le sud. Les petits agriculteurs n'ont pas les moyens de modifier le statu quo local imposé par les acteurs plus puissants en matière de délimitation des territoires agricoles et pastoraux. N'ayant pas de troupeaux, ils doivent tout de même accepter la délimitation de pistes à bétail qui empêchent l'agrandissement de leur champ en saison des pluies.

Si la catégorie des acteurs «perdants " n'est pas fédérée par des intérêts communs conscients, celle des acteurs "gagnants» a su trouver un compromis pour promouvoir ses différents objectifs, à la fois agricoles et pastoraux. En ce sens, ce sont les acteurs «gagnants » qui décident de la «mise en territoire » et qui président aux destinées de la région.

\section{Des perspectives incertaines}

L'ensemble de la région cotonnière se situe dans une phase de transition encore inachevée : la fermeture du front pionnier ne signifie pas la fin des recompositions régionales. Dans un contexte de saturation foncière, l'Ouest du Burkina apparaît en crise. L'étymologie grecque du mot (krisis, le choix) nous rappelle que plusieurs voies pour l'aménagement du territoire régional restent ouvertes. Comme ailleurs en Afrique de l'Ouest, les choix politiques depuis l'indépendance s'orientent plutôt vers une intégration plus aboutie de l'agriculture et de l'élevage grâce à une sédentarisation des transhumants. Mais peu d'expériences ont été concluantes de ce point de vue [Boutrais, 1990]. Il faut donc élargir la réflexion à d'autres formes d'intégration de l'agriculture et de l'élevage. Les complémentarités régionales entre la zone sahélienne au nord et les zones sahélo-soudaniennes au sud méritent d'être repensées.

\section{Quels scénarios possibles?}

La question de la fin des logiques d'organisation spatiale actuelles et de leur remplacement reste posée. La «mise en territoire» en cours peut donner lieu à différentes configurations locales et régionales. Nous en esquisserons trois dans le cadre de cette réflexion. L'évolution vers l'une ou l'autre dépendra en grande partie des compromis et des choix effectués au sein de la catégorie des acteurs «gagnants».

Un premier scénario envisageable est le maintien du statu quo entre les acteurs « gagnants ». La « mise en territoire » déboucherait sur une transition inachevée. Une transhumance nord-sud se maintiendrait, mais deviendrait de plus en plus marginale. Elle serait contrôlée par de grands éleveurs peuls qui, en cultivant des attaches sociales fortes sur la base du réseau des djatigui, tenteraient tant bien que 
mal de garder des territoires de transit (pistes à bétail et quelques pâturages) dans les terroirs du bassin cotonnier. Les agro-éleveurs continueraient de garder la mainmise sur les territoires locaux en privilégiant la culture du coton. Ils tenteraient de trouver des solutions, en coordination avec les agro-pasteurs, pour faire pâturer leurs troupeaux plus au sud, tout en les gardant une partie de l'année auprès de leur exploitation, dans des territoires pastoraux réduits au minimum. La pression locale sur les acteurs «perdants » serait très forte. Deux possibilités seraient alors envisageables. De nouvelles formes de régulation, notamment foncière, seraient trouvées. Les tensions entre acteurs demeureraient fortes, mais ne dégénéreraient pas en conflits incontrôlables. À ces conditions, la cohabitation des différentes activités pourrait, bon gré mal gré, se prolonger à l'échelle locale. Un équilibre entre besoins pastoraux et agricoles résulterait de la « mise en territoire ». Dans une deuxième hypothèse, aucune solution viable ne répondrait à la pression foncière et à la pression démographique. Les conflits se multiplieraient sur la délimitation et l'appropriation des territoires entre acteurs «gagnants » et «perdants », ainsi qu'entre petits agriculteurs et petits éleveurs au sein de la catégorie des acteurs «perdants » (dégâts des animaux dans les champs qui obstruent leur passage, diminution du nombre de pâturages). Ces conflits empêcheraient alors toute intégration de l'élevage et de l'agriculture, et compromettraient la stabilité de la région.

Une autre possibilité est celle d'une fragmentation de l'espace régional. Au sein de la catégorie des acteurs «gagnants », les agro-pasteurs ne garderaient pas suffisamment de pouvoir pour maintenir des territoires pastoraux de transit dans le bassin cotonnier entre la zone sahélienne au nord (pâturages de saison humide) et la zone soudanienne au sud (pâturages de saison sèche). Deux causes sont possibles pour expliquer que les agro-pasteurs seraient in fine les perdants d'une telle «mise en territoire ». Soit les agro-éleveurs exerceraient seuls leur pouvoir sur les territoires locaux; ils réaliseraient une éviction totale des transhumants qui ne pourraient plus circuler. Soit la pression foncière subie par les petits agriculteurs serait trop forte et ils mettraient en culture tous les territoires pastoraux locaux résiduels (pistes et pâturages), empêchant de ce fait la transhumance régionale. Dans ce scénario, les agro-pasteurs devraient se retrancher au nord, dans les territoires sahéliens, ou au sud, dans les territoires soudaniens. Au nord, leur installation permanente (impossibilité de transhumer saisonnièrement) déséquilibrerait les milieux. On ne pourrait qu'assister à la décapitalisation de leur cheptel et à leur paupérisation. Au sud, la pression foncière est forte et le rapport entre agriculteur et éleveur conflictuel depuis longtemps [Bassett, 1988]. Dans ces conditions, une migration massive d'agro-pasteurs est-elle vraiment envisageable ? Dans l'impossibilité de poursuivre les transhumances, ils pourraient, pour sauvegarder leur niveau socio-économique, investir massivement dans l'embouche bovine et renforcer leur intégration dans les filières viande fournissant les marchés urbains et extérieurs. Se poserait alors la question de la viabilité de l'embouche pratiquée à grande échelle. Comment trouver les ressources fourragères suffisantes pour les animaux à l'engraissement? Dans ce scénario, les territoires du bassin cotonnier 
seraient contrôlés par les agro-éleveurs. Ils mettraient en place un système d'agroélevage autonome, circonscrit au bassin cotonnier. Les mobilités des troupeaux seraient uniquement locales, les pressions sur le milieu très fortes. Dans des territoires où la densité de population peut dépasser 60 habitants $/ \mathrm{km}^{2}$, des cycles régressifs (manque de pâturage, baisse des rendements agricoles faute d'une véritable intégration agriculture/élevage) pourraient alors se mettre en place [Dugué, 2004]. Une échappatoire serait une intensification des productions (vivrières, commerciales, cultures fourragères...) qui permettrait d'augmenter les rendements et de peut-être relâcher la pression foncière ; malgré les tentatives dans ce sens, aucune expérience n'est pour le moment concluante dans l'Ouest du Burkina. Pour les agro-éleveurs, l'élevage, qui serait désormais entre leurs mains, jouerait le rôle d'amortisseur des soubresauts de la filière cotonnière (boums suivis de crises [Renaudin, 2010]).

Le dernier scénario proposé est une variante, une version plus aboutie de la «mise en territoire » par les agro-éleveurs. Si le rapport de force va jusqu'au bout et que les grands éleveurs peuls échouent dans leur reconversion, ils seront forcés de quitter la région Ouest. Les agro-éleveurs autochtones et mossis contrôleraient totalement les territoires et les organiseraient selon leurs intérêts. Les troupeaux grandissant, et les milieux se dégradant à cause de mobilités trop restreintes, les agro-éleveurs du bassin cotonnier pourraient alors décider d'envoyer de nouveau leurs troupeaux en transhumance au nord et au sud. Les bêtes seraient alors conduites par des bergers salariés ou par les fils des propriétaires. Une organisation nord-sud des territoires de l'élevage se mettrait donc de nouveau en place. En apparence, la transition s'achèverait par un retour au point de départ, l'organisation de l'espace régional étant similaire à celle qui prévalait auparavant. Mais ces espaces seraient appropriés de manière plus décisive par les agro-éleveurs qui contrôleraient ainsi à leur profit des territoires réticulaires d'échelle régionale.

\section{Conclusion}

L'évocation de ces scénarios d'évolution des systèmes d'élevage peut sembler bien décalée par rapport à la situation présente ; elle présente l'avantage d'attirer l'attention sur les incertitudes d'une période de transition sans point d'équilibre. Face à la complexité des contradictions présentes, le risque est grand que les pouvoirs publics ne prennent pas d'initiatives majeures pour contrôler les évolutions spontanées. Alors que l'objectif des politiques publiques devrait être d'accompagner les transitions et de prévoir les évolutions souhaitables, la difficulté est telle que la facilité de ne rien entreprendre peut l'emporter.

La question centrale demeure celle de la sécurisation de pratiques d'élevage qui restent compatibles avec l'évolution générale de la région, dominée par les logiques agricoles. Sous sa forme actuelle, la mobilité généralisée semble vouée à rencontrer des difficultés grandissantes. Il faut donc innover et proposer des schémas d'aménagement aboutissant à des réorientations fortes des pratiques sociales et économiques; une voie possible pourrait être d'encourager des spécialisations 
complémentaires, renouvelant les bases des échanges entre les zones bioclimatiques. Le nord garderait des effectifs réduits, compatibles avec les contraintes naturelles ; il se spécialiserait en pays naisseur, fournissant les zones agricoles en bétail pour la culture attelée. La zone à vocation agricole tirerait un meilleur parti d'un élevage réduit en nombre mais valorisé par les débouchés urbains par la généralisation de l'embouche. Ce modèle présente une rupture forte par rapport aux pratiques actuelles, mais il ouvre des perspectives pour des interventions spécifiques à chaque zone et propose un schéma global d'aménagement à l'échelle régionale.

\section{Bibliographie}

Augusseau X. [2008], Évolution des systèmes agro-pastoraux dans le sud-ouest du Burkina Faso, interactions et dynamiques territoriales, thèse de géographie, Université Paul Valéry Montpellier-3, $314 \mathrm{p}$.

Bassett T. [1988], "The Political Ecology of Peasant-Herder Conflicts in Northern Ivory Coast", Annals of the Association of American Geographers, vol. 78, nº 3, p. 453-472.

Benort M. [1979], Le Chemin des Peuls du Boobola, Paris, Orstom, 208 p.

Bernardet P. [1999], «Peuls en mouvement, Peuls en conflits en moyenne et haute Côte d'Ivoire, de 1950 à 1990 », in Botte R., Boutrais J., Schmitz J., (dir.), Figures peules, Paris, Karthala, p. 408-444.

BoutRais J. [1990], «Derrière les clôtures... Essai d'histoire comparée des ranchs africains », in Bernus E. Poulloon F. (dir.), "Sociétés pastorales et développement », Cahiers des sciences humaines, vol. 26, 1-2, p. 73-95.

Boutrais J. [1994], « Pour une nouvelle cartographie des Peuls », Cahiers d'études africaines, vol. $34, n^{\circ} 133-135$, p. 137-146.

Boutrais J. [1988], Des Peuls en savanes humides, Paris, Orstom éditions, 387 p.

Cesaro J.-D., Magrin G., Ninot O. [2010], Atlas de l'élevage au Sénégal, Montpellier, Paris, Cirad, ATP Icare, Prodig, Université Paris-1, 32 p.

Diallo Y. [1997], Les Fulbe du Boobola, Cologne, Rüdiger Köppe, 240 p.

Dongmo A.L., Duamen P., Vall E., Koussou M.O., Coulibaly D., Lossouarn J. [2007], «L'espace est fini! Vive la sédentarisation? », Quatorzièmes rencontres autour des recherches sur les ruminants, Paris, Institut de l'élevage, p. 153-160.

Dugue P., Vall E., Lecomte P., Klein H.D., Rollin D. [2004], «Évolution des relations entre l'agriculture et l'élevage dans les savanes d'Afrique de l'Ouest et du Centre. Un nouveau cadre d'analyse pour développer de nouveaux modes d'intervention et favoriser les processus d'innovation », Oléagineux, corps gras, lipides, vol. 11, nº 4, p. 268-276.

Gallais J. [1984], Hommes du Sahel, Paris, Flammarion, 289 p.

Gautier D., Ankogui-Mpoko G.-F., Reounodj F., Nuoya A., Seignobos C. [2005], « Agriculteurs et éleveurs des savanes d'Afrique centrale : de la coexistence à l'intégration territoriale », L'Espace géographique, vol. 2005/3, tome 34, p. 223-236.

Gonin A. [2011], Dynamiques de l'élevage et recomposition de l'Ouest du Burkina, des espaces aux territoires de l'élevage, Lémoire de master 2, université Paris-1, 92 p.

Hoffmann O. [1985], Pratiques pastorales et dynamique du couvert végétal en pays lobi, Paris, Orstom, $355 \mathrm{p}$. 
Homewood K. [2008], Ecology of African Pastoralist Societies, Oxford, Athens, Pretoria, James Currey, Ohio University press, Unisa Press, 292 p.

Jullien F. [2006], « Nomadisme et transhumance, chronique d'une mort avancée ou voie d'un développement porteur. Enjeux, défis et enseignements tirés de l'expérience des projets d'hydraulique pastorale au Tchad ? », Afrique contemporaine, vol. 2006/1, n 217, p. 55-75.

Lericollais A. (dir.) [1999], Paysans sereer, Paris, IRD, 668 p.

Magrin G. [2001], Le Sud du Tchad en mutation, Saint-Maur-des-Fossé, Cirad, Sépia, 470 p.

Marty A., Bonnet B., Guibert B. [2006], « La mobilité pastorale et sa viabilité », Paris, Iram, Note thématique $\mathrm{n}^{\circ} 3,4 \mathrm{p}$.

Pare L., Tallet B. [1999], « D'un espace ouvert à un espace saturé. Dynamique foncière et démographique dans le département de Kouka », Espace, populations et sociétés, $\mathrm{n}^{\circ} 1$, p. 83-92.

Pelissier P. [1978], «L'arbre dans les paysages agraires de l'Afrique noire », in Etudes géographiques offertes à Louis Papy, Bordeaux, Institut de Géographie de Bordeaux-3, CEGET, MSHA, p. 27-32.

Pourtier R. [2005], «Les âges de la territorialité » in Antheaume B., GiRaut F., Le Territoire est mort, vive les territoires! Paris, IRD Éditions, p. 39-46.

RENAUDiN C. [2010], « Les riches heures et l'avenir incertain de la culture cotonnière en Afrique de l'Ouest et du Centre », EchoGéo, no 14, 13 p. : http://echogeo.revues.org/11955 (page consultée le 15 septembre 2011).

Retaille D. [1993], «Afrique, le besoin de parler autrement qu'en surface », in «Les apories $\mathrm{du}$ territoire », Cahiers d'espaces temps, ${ }^{\circ}$ 51-52, p. 52-62.

SAVONNET G. [1959], « Un système de culture perfectionnée, pratiqué par les Bwaba Bobo-Oulé de la région de Houndé (Haute-Volta) », Bulletin de l'IFAN, 31, série B, 3-4, p. 424-458.

Savonnet G. [1968], Atlas de Haute Volta: carte provisoire des densités de population, Ouagadougou, Centre voltaïque de la recherche scientifique, $16 \mathrm{p}$.

Seignobos C., Thys E. [1998], Des taurins et des hommes, Paris, Orstom, 399 p.

TALLET B. [2007], À l'arrière des fronts pionniers. Recompositions territoriales dans l'Ouest du Burkina-Faso et dans le Sud du Veracruz (Mexique), HDR, université Paris 1, 206 p.

Tersiguel P. [1995], Le Pari du tracteur. La modernisation de l'agriculture cotonnière au Burkina-Faso, Paris, Orstom éditions, 280 p.

Vall E., Dugue P., Blanchard M. [2006], «Le tissage des relations agriculture. Élevage au fil du coton », Cahiers agricoles, vol. 15, $\mathrm{n}^{\circ} 1$, p. 72-79. 